Originalien

Schmerz 2020 34:319-325

https://doi.org/10.1007/s00482-020-00453-1

Online publiziert: 3. März 2020

(c) Der/die Autor(en) 2020

N. Best ${ }^{1,2} \cdot$ D. Loudovici-Krug ${ }^{1,3} \cdot$ P. Zeits $\cdot$ W. Meißner ${ }^{4,5} \cdot$ U. C. Smolenski ${ }^{1,4}$

${ }^{1}$ Institut für Physiotherapie, Universitätsklinikum Jena, Jena, Deutschland

${ }^{2}$ Institut für Physiotherapie, Posture and Motion Group, Universitätsklinikum Jena, Jena, Deutschland

${ }^{3}$ Forschungsberatungsstelle für Manuelle Medizin, gefördert durch die Deutsche Stiftung für Manuelle Medizin, Jena, Deutschland

${ }^{4}$ Interdisziplinäre Multimodale Schmerztherapie - Tagesklinik, Universitätsklinikum Jena, Jena, Deutschland

${ }^{5}$ Klinik für Anästhesie und Intensivmedizin, Universitätsklinikum Jena, Jena, Deutschland

\title{
Klinische Anwendung des Bregmatests
}

\section{Punktprävalenz an Gesunden, Nichtschmerzpatienten und chronischen Schmerzpatienten sowie Änderung der Testergebnisse während einer interdisziplinären multimodalen Schmerztherapie}

tinearbeit als tauglich erwiesen [4]. Dabei wird das Bregma mechanisch mittels Fingerkratzen fazilitiert. Das Bregma ist der Punkt der Verbindung zwischen Pfeilnaht und Kranznaht und Residuum der großen Fontanelle. Der Untersuchte wird nun gebeten, den vormals fazilitierten Punkt Richtung Decke zu führen. Dabei sollen die Füße den Boden nicht verlassen. Als physiologisch gelten Bewegungen, mit exakt vertikal nach oben geführtem Bregma. Als pathologisch gelten Bewegungen, bei denen der Kopfnach hinten oder vorn bewegt wird und damit das Bregma nicht optimal in die Vertikale gelangen kann [3]. Bei beiden Varianten kommen Parakinesen als beiläufige, aber nicht notwendige Bewegungsanteile hinzu. In der folgenden Aufstellung sind die Bregmatestwerte aufgeführt. Folge der Bregmavertikalisierung sind eine aktive Haltungskorrektur der Schwerkraft entgegen und konsekutiv die Abflachung der Wirbelsäulenschwünge, wenn diese vorhanden waren. In 0 Abb. 1 sind schematisch die physiologischen Haltungskorrekturen dargestellt. - Tab. 1 zeigt die Graduierung in Abstufungen nach der definierten Einteilung des Bregmatests. häufig Pathologien bei Bewegungsausführung in unterschiedlichen Untersuchungsgruppen auftreten. Dabei wurden Gesunde, Patienten, die keine Schmerzstörung im Sinne der ICD-10 F45.41 als Diagnose führen, und Patienten, die diese Diagnose tragen, untersucht.

\section{Hintergrund und Fragestellung}

Der Bregmatest gilt als Test zur Abschätzung der allgemeinen sensomotorischen Fähigkeiten im Stehen und hat sich als reliables Instrument für die tägliche Rou-

\section{Tab. 1 Bregmatestgraduierungen}

\begin{tabular}{|l|l|l|l|}
\hline \multicolumn{2}{|l|}{ Ausführung } & \multicolumn{2}{l}{ Bregmatestgrad und Ausführung } \\
\hline $1 . x$ & $\begin{array}{l}\text { Physiologisch } \\
\text { Bregma korrekt }\end{array}$ & 1.0 & Ohne Parakinesen \\
\hline & 1.1 & Mit Parakinesen im Schultergürtel \\
\hline & 1.2 & Mit Parakinesen im orofazialen System \\
\hline \multirow{2}{*}{ vertikalisiert } & 1.3 & Mit Parakinesen im Schultergürtel und orofazial \\
& $\begin{array}{l}\text { Pathologisch } \\
\text { Bregma nicht korrekt }\end{array}$ & 2.0 & Ohne Parakinesen \\
\hline vertikalisiert & 2.1 & Mit Parakinesen im Schultergürtel \\
& 2.2 & Mit Parakinesen im orofazialen System \\
& 2.3 & Mit Parakinesen im Schultergürtel und orofazial \\
\end{tabular}




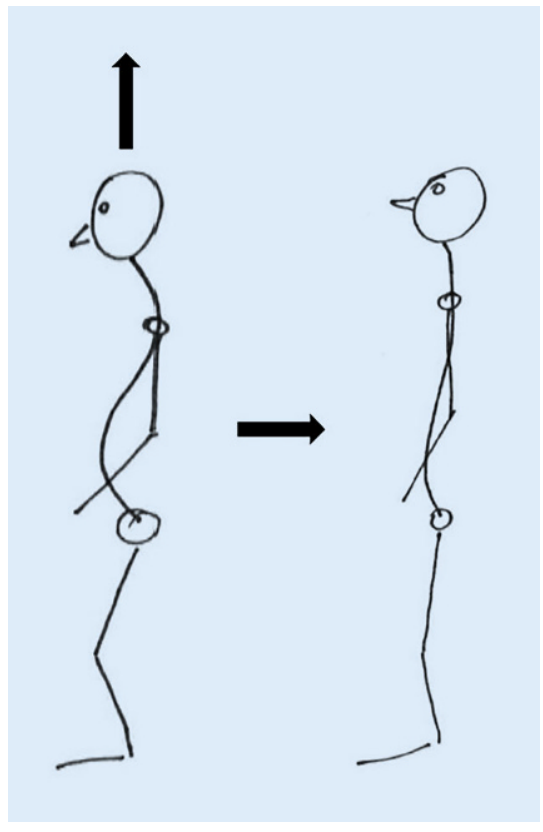

Abb. 1 \& Physiologische Bewegungen beim Bregmatest

Es ist anzunehmen, dass Störungen motorischer Stereotype, auch in der gesunden Bevölkerung weit verbreitet sind. Neben erkrankten zeigen auch gesunde Menschen Haltungs- und Bewegungsstörungen [9, 17, 19]. Für Schmerzpatienten sind, zumindest bei ausgewählten Erkrankungsentitäten, Körperwahrnehmungsstörungen beschrieben [5]. Dabei stellen Körperwahrnehmungsschulungen, Visualisierungen und kognitive Techniken in der interdisziplinären multimodalen Schmerztherapie etablierte Methoden zur Beeinflussung solcher Funktionsdefizite dar [15].

In die Kategorie der Körperwahrnehmungs- und Koordinationstests gehört auch der Bregmatest. Es stellt sich die Frage, ob es Unterschiede in der Prävalenz pathologischer Bregmatests zwischen chronischen Schmerzpatienten im Vergleich zu Patienten ohne diagnostiziertes chronisches Schmerzsyndrom mit somatischen und funktionellen Störungen sowie Gesunden gibt.

\section{Studiendesign und Untersu- chungsmethoden}

Im Rahmen dieser Querschnittsstudie wurden nach Zustimmung zur Studien-

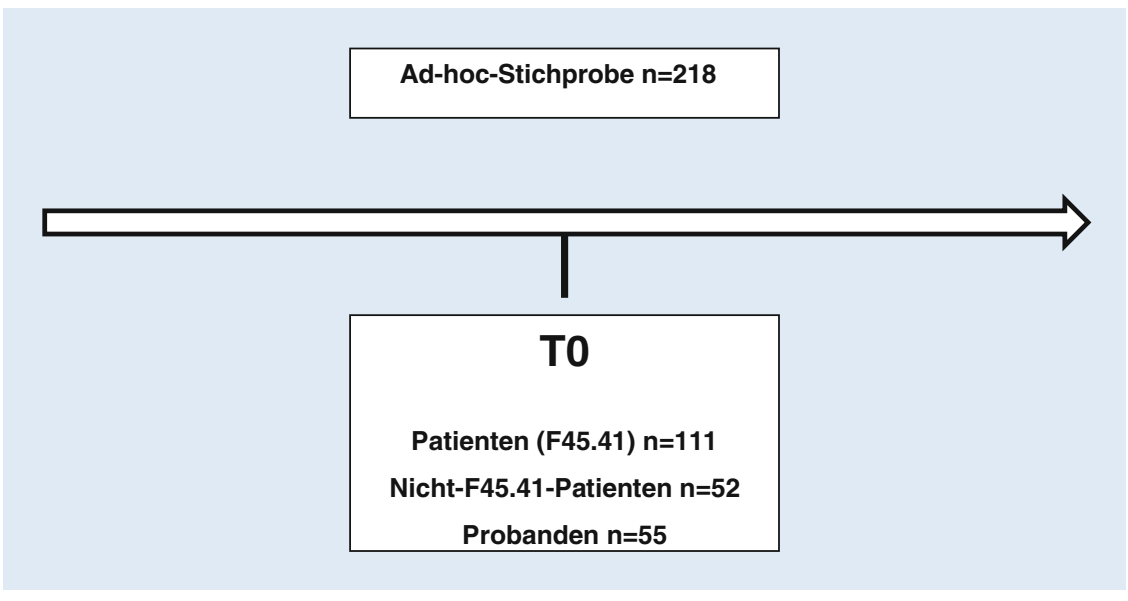

Abb. $2 \Delta$ Ad-hoc-Stichprobe T0, $n=218$

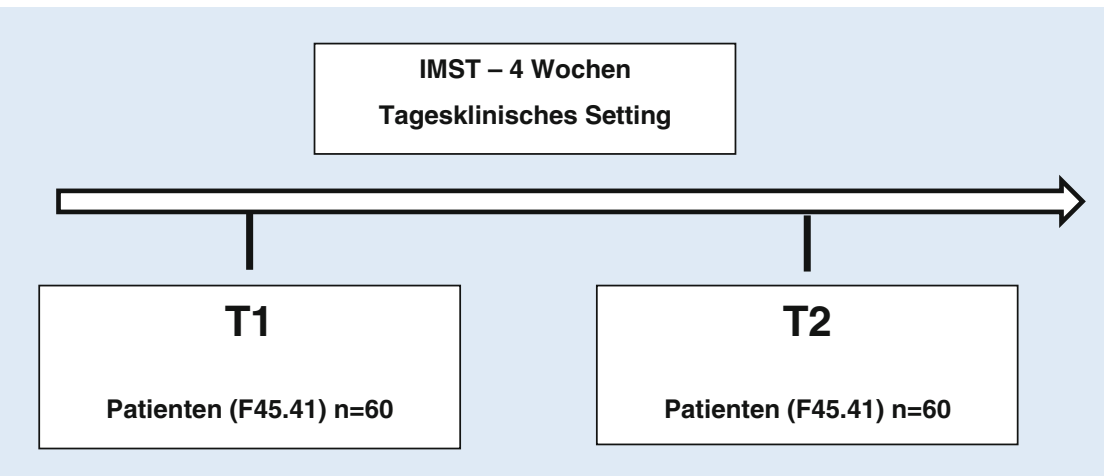

Abb. $3 \Delta$ Zeitlicher Studienablauf T1-T2, $n=60$

teilnahme Probanden und Patienten untersucht.

Initial wurde die Punktprävalenz bei einer Stichprobe im Umfang von $n=218$ geprüft. Die Patientenpopulation wurde in eine Gruppe von Menschen mit der Diagnose „Chronische Schmerzstörung mit somatischen und psychischen Faktoren" (ICD-10 F45.41), eine Gruppe Patienten ohne diese Diagnose sowie Gesunde aufgeteilt. Die Diagnose F45.41 wurde in der eigenen Einrichtung durch erfahrene KollegInnen in Teamarbeit gestellt. Unter anderem wurde der Deutsche Schmerzfragebogen als Assessment genutzt. Das Team bestand aus Ärzten aus dem Bereich der Anästhesiologie und der physikalischen und rehabilitativen Medizin sowie erfahrenen Psychologen mit Fortbildung in psychologischer Schmerztherapie.

In einem zweiten Teil der Untersuchungen konnten $n=60$ chronische Schmerzpatienten, die im Rahmen ei- ner interdisziplinären multimodalen Schmerztherapie behandelt wurden, im Verlauf beobachtet werden. Die tagesklinische Behandlung dauerte standardisiert 4 Wochen. An 20 Behandlungstagen wurden gemäß üblichen Empfehlungen der Schmerzgesellschaften neben bewegungstherapeutischen Konzepten auch kognitiv-behaviorale Ansätze einschließlich Entspannungstherapien und edukativer Inhalte angewendet [18]. Dabei wurde ein standardisiertes Rahmenprogramm in der Gruppe mit individualisierten Inhalten und relativ wenigen Einzelgesprächen und -therapien durchgeführt.

Eine grafische Aufarbeitung des Studienablaufs zeigen $\bullet$ Abb. 2 und 3. 


\section{Ergebnisse}

\section{Deutliche Unterschiede pathologischer Bregmatests in der Ad-hoc-Stichprobe}

Zwischen den 3 untersuchten Gruppen gab es deutliche Unterschiede. Betrachtet man die Bregmatestwerte von 1.x, also $1.0,1.1,1.2$ und 1.3, als physiologisch bzw. physiologisch mit kompensatorischen Bewegungsmustern und 2.x in Analogie zu 1.x als pathologisch, dann fällt eine deutliche Häufung pathologischer Bregmatestgrade bei den chronischen Schmerzpatienten auf.

\section{Patienten mit chronischem Schmerzsyndrom zeigen deutlich häufiger auffällige Bregmatests als die anderen untersuchten Gruppen}

Einen signifikanten Unterschied zwischen der Gruppe der Patienten, die nicht an einem chronischen Schmerzsyndrom leiden, und den gesunden Probanden zeigte sich nicht. Ca. jeder siebte Patient bzw. Proband vertikalisiert sein Bregma gemäß der Technikbeschreibung nach Aufforderung nicht korrekt. Bei Patienten mit der Diagnose F45.41 traten bei über $50 \%$ der untersuchten Patienten $\mathrm{Pa}$ thologien in der Bewegungsausführung im Rahmen des Bregmatests auf.

Im zweiten Teil der Arbeit, bei dem ausschließlich Patienten mit der Diagnose F45.41 im Verlauf einer interdisziplinären multimodalen Schmerztherapie beobachtet wurden, zeigte sich in Bezug auf den Bregmatest ein deutlicher „benefit“. Zum Untersuchungszeitpunkt T1 waren ebenfalls über $50 \%$ der untersuchten Personen sensomotorisch nicht ausreichend kompetent, um den Bregmatest physiologisch auszuführen. Nach Abschluss unseres Therapieprogramms zeigten sich dagegen lediglich noch ca. $30 \%$ auffällig.

Eine grafische Aufarbeitung der Daten kann - Abb. 4 und 5 entnommen werden.

Weiterhin konnten wir nachweisen, dass in der Verlaufsbeobachtung initial lediglich nicht ganz ein Drittel der Patienten und zum Zeitpunkt T2 nunmehr $50 \%$ aller Untersuchten einen fehlerfrei-

Schmerz 2020 -34:319-325 https://doi.org/10.1007/s00482-020-00453-1

(c) Der/die Autor(en) 2020

N. Best · D. Loudovici-Krug · P. Zeits · W. Meißner · U. C. Smolenski

Klinische Anwendung des Bregmatests. Punktprävalenz an Gesunden, Nichtschmerzpatienten und chronischen Schmerzpatienten sowie Änderung der Testergebnisse während einer interdisziplinären multimodalen Schmerztherapie

\section{Zusammenfassung}

Hintergrund. Der Bregmatest (BT) als Untersuchung sensomotorischer Defizite wurde bislang nicht in der Breite angewandt. Er gilt als ein Test zur Darstellung allgemeiner sensomotorischer Fähigkeiten im Stand. Schmerzpatienten zeigen häufig Körperwahrnehmungs- und Bewegungsstörungen. Ziel der Arbeit. Gibt es Unterschiede in der Punktprävalenz beim Bregmatest bei Patienten (mit und ohne die Diagnose F45.41) bzw. Gesunden. In einem zweiten Teil wird die Entwicklung pathologischer BTWerte im Rahmen einer interdisziplinären multimodalen Schmerztherapie (IMST) beobachtet.

Material und Methoden. Punktprävalenz pathologischer Testergebnisse in 3 Gruppen, insgesamt $n=218$. Verlaufsbeobachtung im Rahmen einer IMST zu Beginn und am Ende eines tagesklinischen Settings, $n=60$. Ergebnisse. Gesunde und "Nicht-F45.41Patienten" zeigen $<15 \%$ Häufigkeit des
Auftretens pathologischer Bregmatestwerte. Bei „F45.41-Patienten“ Iag die Häufigkeit bei $>50 \%$. Im Rahmen einer IMST ließen sich die pathologischen Bewegungsausführungen auf ca. $33 \%$ absenken.

Diskussion. Gehäufte Körperwahrnehmungsstörungen sind für chronische Schmerzpatienten bekannt. Der Bregmatest ist in der Lage, zumindest einen Teil der Patienten, die sensomotorische Defizite haben, zu detektieren. Chronische Schmerzpatienten unterscheiden sich hier signifikant von Gesunden oder anderen Patientengruppen. Die Defizite in der Ansteuerbarkeit myofaszialer Gewebe (Koordination) sind auch im Rahmen einer vierwöchigen IMST besserbar.

\section{Schlüsselwörter}

Schmerztherapie · Klinischer Test · Bregmatest $\cdot$ Sensomotorische Kontrolle . Haltungskontrolle

\section{Clinical application of the Bregma test. Point prevalence in healthy, pain-free and chronic pain patients and change of test results during interdisciplinary multimodal pain therapy}

\section{Abstract}

Background. The Bregma test as an investigation of sensorimotor deficits has not yet been broadly applied. It is considered to be a test for the presentation of general sensorimotor abilities in a standing position. Pain patients often show disorders in physical perception and movement.

Objective. Are there differences in the point prevalence of the Bregma test in patients (with and without diagnosis F45.41) or healthy persons? In a second part the development of pathological Bregma test values was observed in the context of an interdisciplinary multimodal pain therapy (IMST).

Material and methods. Point prevalence of pathological test results in 3 groups, total $n=218$. Monitoring of the course of an IMST at the beginning and end of a day clinic setting, $n=60$.
Results. Healthy and "non-F45.41" patients showed $\mathrm{a}<15 \%$ frequency of pathological Bregma test results. In "F45.41 patients" the frequency was $>50 \%$. Within the framework of an IMST the pathological movements could be reduced to approximately $33 \%$.

Conclusion. Increased body perception disorders are known in chronic pain patients. The Bregma test is able to detect at least some of the patients with sensorimotor deficits. Chronic pain patients significantly differ from healthy or other patient groups. The deficits in the controllability of myofascial tissue (coordination) can also be improved during a 4-week IMST.

Keywords

Pain therapy - Clinical trial - Bregma test . Sensorimotor control · Posture control 


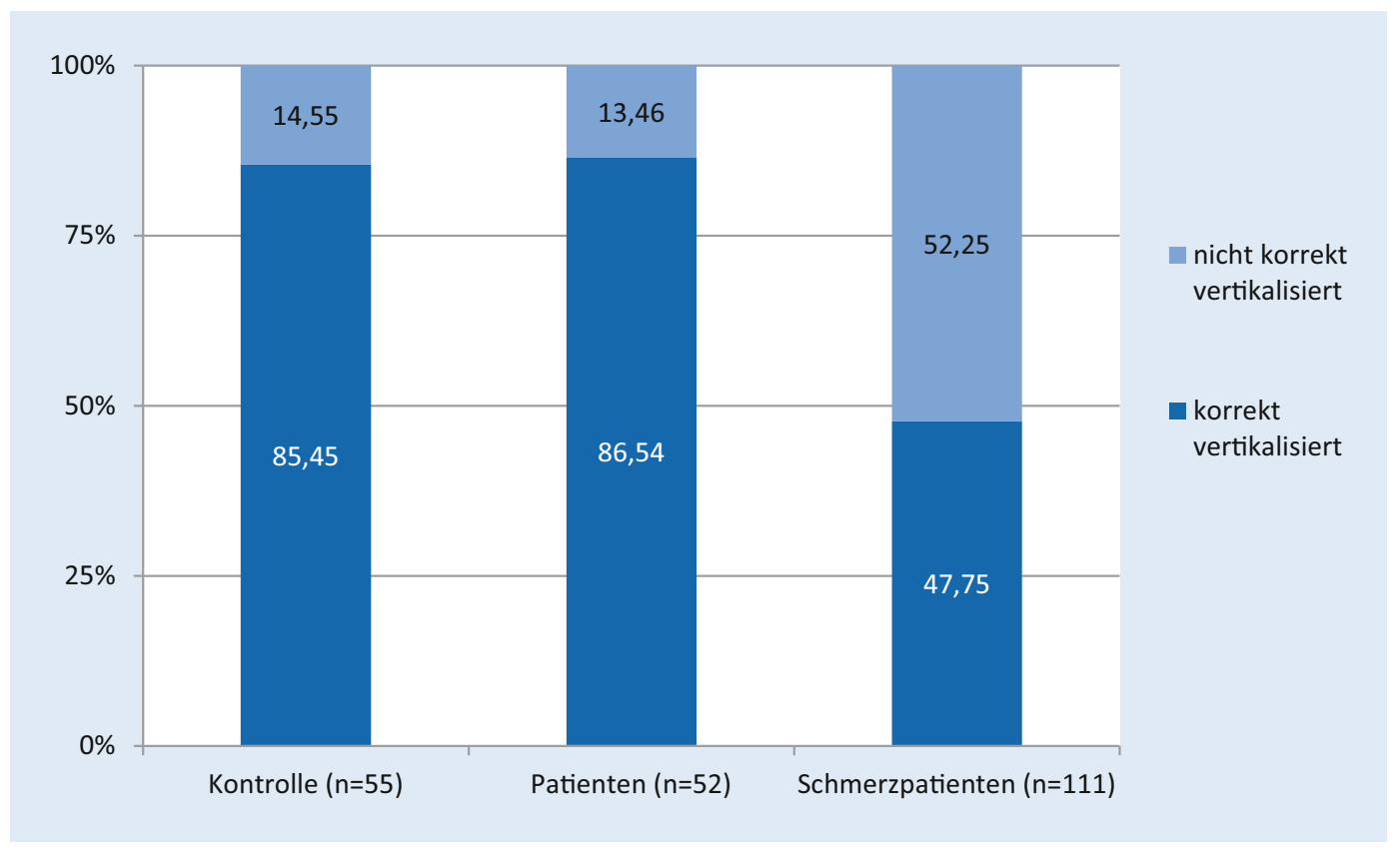

Abb. $4<$ Verteilung der Bregmatestergebnisse nach Studienpopulationen

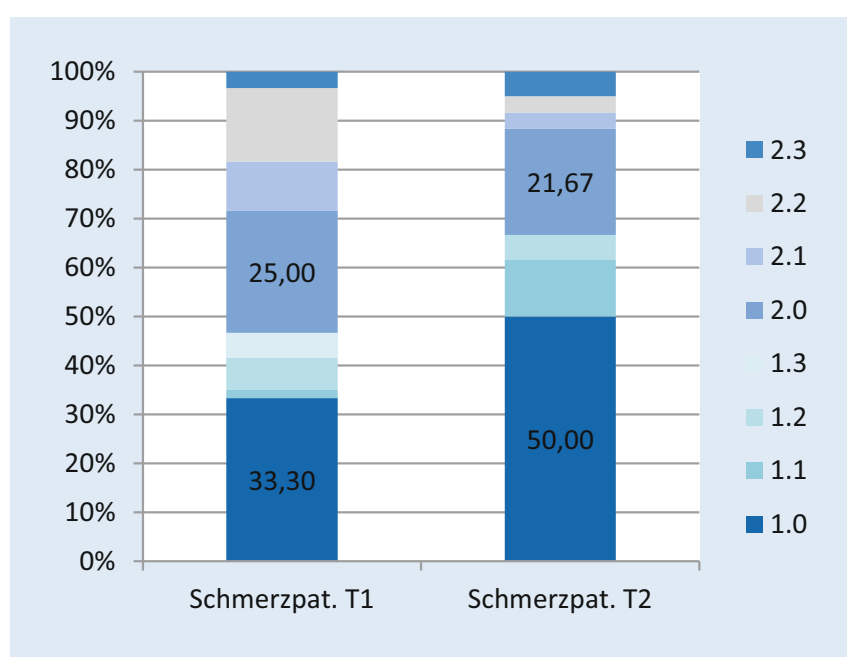

Abb. 5 \ Detaillierte Aufstellung der Bregmatestergebnisse der Schmerzpatienten $(n=60)$ im Verlauf

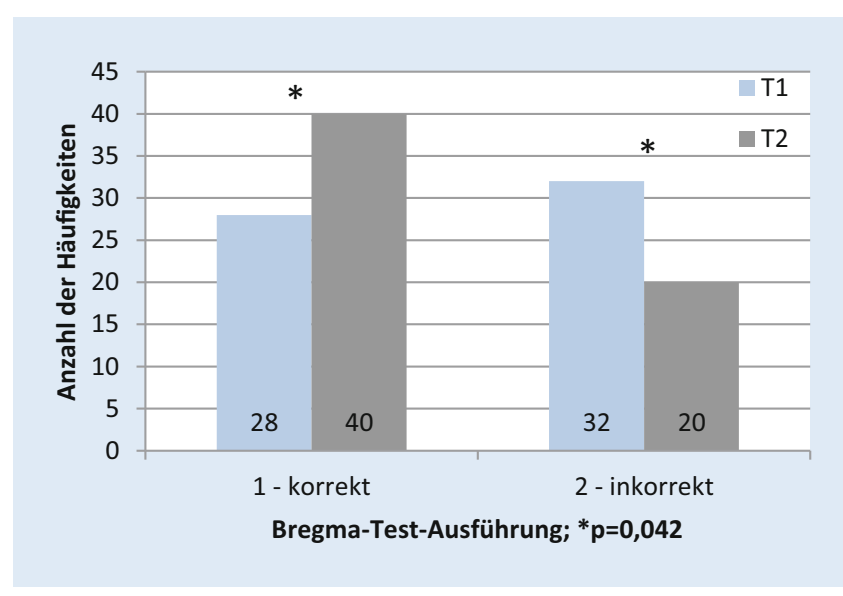

Abb. 6 \ Veränderung der Gesamtheit der Bregmatestausführungen der Schmerzpatienten en Bregmatest ausführen konnten. Der bei Weitem größte Anteil dieser nun sensomotorisch optimierten Patienten rekrutierte sich aus der 1.x-Gruppe. Trotz der Tatsache, dass die absolute Anzahl an fehlerhaft ausgeführten Bregmatests sank, konnten wir keine signifikante Veränderung der maximal pathologisch ausgeführten Variante [2,3] feststellen.

\section{Im Rahmen einer IMST lassen sich sensomotorische Defizite mindern}

Sowohl die Veränderungen korrekt bzw. inkorrekt ausgeführter Bregmatests von
Zeitpunkt T1 zu T2 als auch die Gesamtveränderungen zwischen T1 und T2 erwiesen sich als signifikant. Die Veränderung der Werte wird in $\bullet$ Abb. 6 und 7 dargestellt.

Bei der detaillierten Analyse der Veränderungen von T1 zu T2 konnten 27 Patienten detektiert werden, die sich beim Bregmatest verbessern konnten. Eine Verbesserung des Bregmatestwerts von 2.x hin zu 1.x zeigte sich bei 13 Studienteilnehmern, eine Verschlechterung der Werte bei 8 Patienten. Dabei kam es in einem Fall zu einer Veränderung von einem Test $1.0 \mathrm{zu}$ einem Test $2.0 \mathrm{im}$ Rahmen der Verlaufsbeobachtung.

\section{Diskussion}

Die erhobenen Daten zeigen, dass der Bregmatest bei Patienten, die unter der Diagnose F45.41 leiden, deutlich häufiger pathologisch ausfällt, als bei den als Kontrollgruppen untersuchten Studienpopulationen. Wie bereits 2012 von Bißwanger-Heim dargestellt wurde, sind Körperwahrnehmungsstörungen bei Menschen mit chronischer Schmerzstörung häufiger anzutreffen [5]. Im vorliegenden Fall 


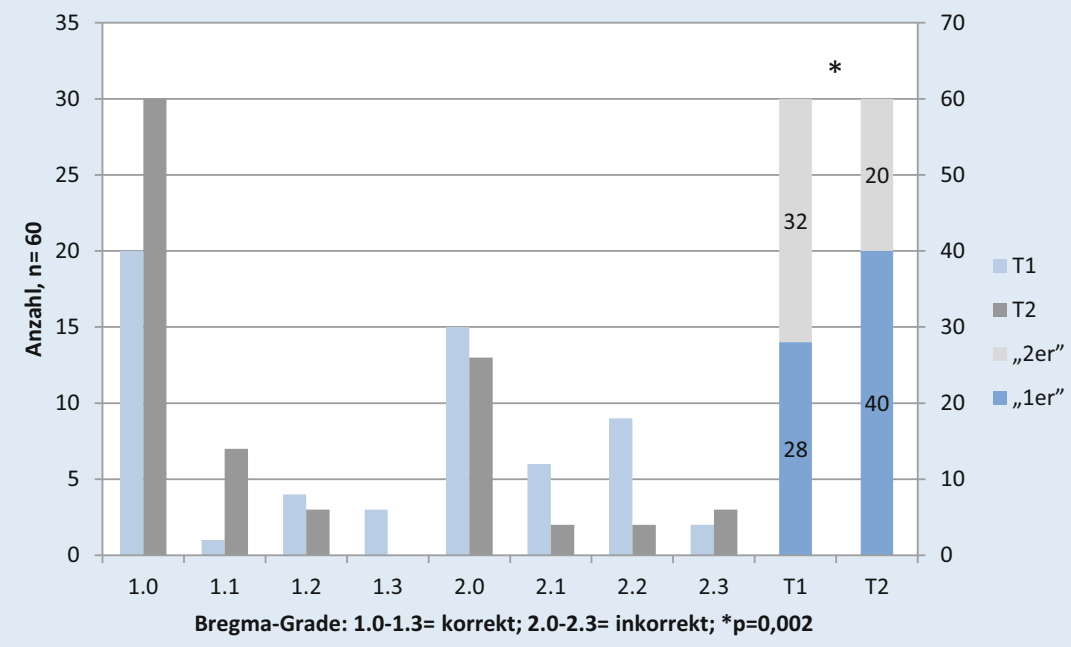

Abb. 7 A Detaillierte Darstellung der Bregmatestveränderungen der Schmerzpatienten

ist die sensomotorische Ansteuerungsfähigkeit ohne adäquate Therapie bei mehr als $50 \%$ der Patienten nachzuweisen. Erstaunlich ist, dass sowohl Gesunde als auch Patienten mit verschiedenen Diagnosen hinsichtlich des Bewegungssystems mit degenerativer Genese keine unterschiedliche Häufung auffälliger Bregmatests zeigen.

Es ließ sich herausarbeiten, dass die übliche Prävalenz pathologischer Bregmatestwerte unter $15 \%$ liegt.

\section{Jeder siebte Nichtschmerzpatient hat einen pathologischen \\ Bregmatest}

Die Autorengruppen um Lauche et al. und Levenig et al. stützen unsere Ansicht $[11,12]$.Zech und Hübscher sprechen davon, dass sensomotorische Schwierigkeiten das Risiko für chronische Schmerzzustände, aber vor allem auch Traumata erhöhen [24].

Die gleichen Autoren berichten gemeinsam mit anderen Kollegen, dass im Rahmen einer Übersichtsarbeit Hinweise gefunden wurden, dass sensomotorisches Training die posturale und neuromuskuläre Fähigkeit verbessern und effektivieren kann [25]. Somit ist nicht abschließend geklärt, ob Menschen mit sensomotorisch defizitären Situationen häufiger dazu neigen, chronische myofasziale Schmerzzustände erleiden zu müssen, oder ob dieses Defizit durch Hemmung der Lebensqualität und der allgemeinen Mobilität gefördert oder gar ausgelöst wird. Aus der täglichen Arbeit mit Patienten und den Erfahrungen der Autoren scheinen beide Kausalzusammenhänge möglich. Unterstützt würde dieses Modell der gegenseitigen Einflussnahme sensomotorischer Defizite und chronischer Schmerzzustände durch die Erkenntnisse, dass bei diesen Patienten unimodale Ansätze, die entweder auf seelisches Schmerzerleben oder körperliche Funktionsfähigkeit abzielen, häufig nicht zum Erfolg führen, wie gedacht. Dies unterstreicht die Wichtigkeit interdisziplinärer multimodaler Therapieansätze, wie sie heute üblich sind [18]. Arnold et al. bestätigen in ihrer 2014 veröffentlichten Arbeit, dass multimodale Therapieansätze in jeden Fall auch zur „Förderung einer positiven Körperwahrnehmung “ eingesetzt werden müssen und geeignet seien [2].

Diese (interprofessionellen) Denkmodelle setzen sich zunehmend auch in anderen, rehabilitativen, Behandlungsansätzen durch [8, 14, 20, 23].

Noeker et al. beziehen die Verbesserung der Körperwahrnehmungen zwar nur auf die Entspannungsverfahren, dennoch kann postuliert werden, dass Menschen, die in der Lage sind, Entspannungsverfahren auch körperlicher Art (bspw. progressive Muskelrelaxation nach Jacobsen) anzuwenden, durchaus eine positive Körperwahrnehmung be- sitzen müssen [16]. Somit könnte es zutreffen, dass eine positive Körperwahrnehmung funktionelle Beschwerden minimieren oder gar vermeiden kann und womöglich die Voraussetzung dafür ist, aktive Entspannungsverfahren gewinnbringend einsetzen zu können. Da Entspannungsverfahren im Rahmen der IMST einen hohen Stellenwert besitzen, wäre es möglich, dass in unserem Setting die Körperwahrnehmung durchaus auch durch diese Art der Therapie verbessert werden konnte.

\section{Auffällige Bregmatests sind nicht gleichbedeutend mit klinisch relevanter Pathologie}

Es stellt sich die Frage, warum Menschen, die sich für gesund halten, sowie Patienten, die nicht unter einem chronischen Schmerzsyndrom nach Definition der ICD-10 F45.41 leiden, dennoch in fast $15 \%$ der Fälle pathologische Bewegungsausführungen zeigen. Dabei ist zu berücksichtigen, dass Funktionspathologien, Kompensationsmuster und sogar Bewegungseinschränkungen häufig für die Betroffenen asymptomatisch bleiben oder gar als „normal“ wahrgenommen werden $[1,13,21,22]$.

Durch unsere Lebensweise werden Bewegungsmuster und -weisen einer Sozialisation unterworfen, auf die die Evolution nicht „vorbereitet“ ist. Nicht nur Unterschiede zwischen den Geschlechtern sind mittlerweile beschrieben [10], sondern auch Modelle, wodurch durch regelmäßiges Üben und Trainieren „neue“, ggf. besondere sensomotorische Fähigkeiten erworben werden können [6].

Oft ist es sogar so, dass funktionelle Störungen (segmentale Dysfunktionen) gar als kompensatorisches Muster notwendig scheinen. Häufig zeigt sich, dass diese in der klinischen Untersuchung auffälligen Befunde zwar erhoben werden können, aber hinsichtlich ihrerklinischen Relevanz gewichtet werden müssen [7]. Mit großer Wahrscheinlichkeit ist dies bei pathologischer Ausführung des Bregmatests auch so. Man könnte gar sagen, dass jeder siebte Untersuchte reflektorisch sensomotorisch inkompetent wurde durch Bewegungsmangel 
und den als Zivilisation bekannten und allseits anerkannten Lebensstil und dies unter den genannten Bedingungen nunmehr „physiologisiert" wird. Aus unserer Sicht kann man aus einem pathologischen Bregmatestbewegungsmuster keinen Krankheitswert ableiten. Allerdings ist $\mathrm{zu}$ prüfen, inwieweit pathologische Bregmatests als Prodrome für die Ausbildung eines chronifizierten Schmerzsyndroms angesehen werden können. Dabei wäre selbstverständlich der pathologische Bregmatest nur ein Ausdruck körperlicher und sensomotorischer Unzulänglichkeiten.

Der Bregmatest ist ein rein subjektiv auszuwertender klinischer Test. In der Arbeit von Best et al. erwies sich dieser Test als ausreichend reliabel [4]. Dennoch besteht Restunsicherheit in der Interpretation der ausgeführten Bewegungen. Es ist also durchaus vorstellbar, dass die nachgewiesenen Verbesserungen, aber auch die Verschlechterungen durchaus durch Fehlinterpretationen verzerrt sein könnten. Dies trifft v. a. auf die einmalig aufgetretene Änderung des Testergebnisses von Grad 1.0 hin zu Grad 2.0 zu.

\section{Schlussfolgerung}

Aus den oben aufgeführten Daten lässt sich schlussfolgern, dass ein pathologischer Bregmatest allein keinen Krankheitswert hat. Wenn hinzu aber chronisch rezidivierende Schmerzen und/ oder deutlich hervortretende Funktionsstörungen kommen, könnte dies ein Hinweis auf die Entwicklung hin zu einem chronischen Schmerzsyndrom sein.

Diesbezüglich lässt sich allgemein empfehlen, die sensomotorische Kompetenz aller Menschen zu optimieren. Was wiederum zur Empfehlung von allgemeiner Bewegung, auch Sport, und Schulung bzw. Training des Bewegungssystems in allen nicht maximalen Facetten führt.

Wir können auch schlussfolgern, dass ein interprofessionelles multimodales Setting im Rahmen einer tagesklinischen Behandlung dazu führen kann, sensomotorische Defizite zu vermindern, selbst wenn dieses nur 4 Wochen dauert. Es ist anzunehmen, dass re- gelmäßige Bewegung, wie oben bereits benannt, eine dauerhafte Verbesserung sensomotorischer Kompetenz bedeuten kann.

\section{Fazit für die Praxis}

Aus Sicht der Autoren zeigt der Bregmatest sensomotorische Auffälligkeiten beim Stehen an. Die übliche Punktprävalenz liegt bei unter $15 \%$. Patienten mit einer chronischen Schmerzstörung mit somatischen und psychischen Faktoren zeigten mit über $50 \%$ eine deutliche Häufung pathologischer Bewegungsmuster im Bregmatest. Patienten mit anderen Diagnosen waren gleich häufig von pathologischen Bewegungen betroffen wie Gesunde. Die körperzentrierten Therapieprinzipien innerhalb einer IMST sind geeignet, die sensomotorische Ansprechbarkeit, die im Rahmen des Bregmatests geprüft wird, zu bessern. Aus Sicht der Autoren ist der Bregmatest als schnell und einfach durchführbarer Screeningtest zur Verlaufsbeobachtung auch innerhalb von kurzen Intervallen geeignet, Veränderungen in der Körperwahrnehmung und -ansteuerung von chronischen Schmerzpatienten abzubilden. Es gilt zu prüfen, ob eine Häufung von pathologischen Bregmamustern auch bei anderen Krankheitsentitäten auftritt.

\section{Korrespondenzadresse}

\section{Dr. med. N. Best}

Institut für Physiotherapie, Universitätsklinikum Jena

Am Klinikum 1, 07747 Jena, Deutschland norman.best@med.uni-jena.de

Funding. Open Access funding provided by Projekt DEAL.

\section{Einhaltung ethischer Richtlinien}

Interessenkonflikt. N. Best, D. Loudovici-Krug, P. Zeits, W. Meißner und U.C. Smolenski geben an, dass kein Interessenkonflikt besteht.

Für diesen Beitrag wurden von den Autoren keine Studien an Menschen oder Tieren durchgeführt. Für die aufgeführten Studien gelten die jeweils dort angegebenen ethischen Richtlinien.

Open Access. Dieser Artikel wird unter der Creative Commons Namensnennung 4.0 International Lizenz veröffentlicht, welche die Nutzung, Vervielfältigung, Bearbeitung, Verbreitung und Wiedergabe in jeglichem Medium und Format erlaubt, sofern Sie den/die ursprünglichen Autor(en) und die Quelle ordnungsgemäß nennen, einen Link zur Creative Commons Lizenz beifügen und angeben, ob Änderungen vorgenommen wurden.

Die in diesem Artikel enthaltenen Bilder und sonstiges Drittmaterial unterliegen ebenfalls der genannten Creative Commons Lizenz, sofern sich aus der Abbildungslegende nichts anderes ergibt. Sofern das betreffende Material nicht unter der genannten Creative Commons Lizenz steht und die betreffende Handlung nicht nach gesetzlichen Vorschriften erlaubt ist, ist für die oben aufgeführten Weiterverwendungen des $\mathrm{Ma}$ terials die Einwilligung des jeweiligen Rechteinhabers einzuholen.

Weitere Details zur Lizenz entnehmen Sie bitte der Lizenzinformation auf http://creativecommons.org/ licenses/by/4.0/deed.de.

\section{Literatur}

1. Angermaier M (2018) Leitfaden Ohrakupunktur. Elsevier, München

2. Arnold B, Brinkschmidt T, Casser H-R et al (2014) Multimodale Schmerztherapie für die Behandlung chronischer Schmerzsyndrome. Schmerz 28:459-472

3. Best N, Best S, Bocker B et al (2017) The Bregmatest (BT) - a screening test for estimating the disturbances of general senso-motoric skills in the deep stabilizing system. Phys Med Rehab Kuror 27:83-86

4. Best $N$, Senftleben $U$, Rottländer-Trier $K$ et al (2019) Reliability and validity of the Bregma-test. Phys Med Rehab Kuror. https://doi.org/10.1055/a1002-0263

5. Bißwanger-Heim T (2012) Körperrepräsentation bei Schmerzerkrankungen. Angew Schmerzther Palliativmed 5:45

6. Gisler-Hofmann T (2008) Plastizität und Training der sensomotorischen Systeme. Schweiz Z Sportmed Sporttraumatol 56:137-149

7. Harke G (2017) Prinzipien manueller Diagnostik. In: Kayser R, Beyer L (Hrsg) Repetitorium Manuelle Medizin/Chirotherapie: Zur Vorbereitung auf die Prüfung der Zusatz-Weiterbildung. Springer, Berlin, Heidelberg, S45-56

8. Hass HG, Rockstroh J, Pech I et al (2018) Medizinisch-beruflich orientierte Rehabilitation (MBOR) in der Onkologie - 2-Jahres-Erfahrungen mit einem standardisierten, multimodalen Therapiekonzept. Phys Med Rehab Kuror 28:341-346

9. Jüngling N, Smolenski UC, Loth D (2004) Untersuchung zur Reliabilität und Validität der dreidimensionalen Funktionsanalyse des Kiefergelenks. Man Med 42:441-448

10. Kirchner B (2018) Geschlechtliche Bewegungsmuster. In: Kirchner B (Hrsg) Bewegungskompetenz:Sportklettern-Zwischen (geschlechtlichem) Können, Wollen und Dürfen. Springer, Wiesbaden, S121-152

11. Lauche R, Cramer H, Haller H et al (2012) My back has shrunk: the influence of traditional cupping on body image in patients with chronic non-specific neck pain. Forsch Komplementmed 19:68-74

12. Levenig CG, Hasenbring MI, Kleinert J et al (2016) Körperbild und Rückenschmerz. Schmerz 30:437-443 
13. Maitland GD (1991) Prinzipien der Techniken. In: Maitland GD (Hrsg) Manipulation der Wirbelsäule. Springer, Berlin, Heidelberg, S134-147

14. Niemier K, Hogrefe H-C (2017) Chronische zervikale Schmerzsyndrome. Vorstellung eines multimodalen interdisziplinären stationären Behandlungskonzepts (ANOA-Konzept). Phys Med Rehab Kuror 27:298-307

15. Niemier K, Seidel W (2009) Funktionelle Schmerztherapie des Bewegungssystems. Springer, Heidelberg

16. Noeker M, Von Rüden U, Staab D et al (2000) Prozesse der Körperwahrnehmung und deren therapeutische Nutzung in der Pädiatrie: Die Weiterentwicklung unspezifischer Entspannungsverfahren zum Training krankheitsspezifischer Beschwerde- und Symptomwahrnehmung. Klin Padiatr 212:260-265

17. Perry J (2003) Ganganalyse - Norm und Pathologie des Gehens. Urban \& Fischer, München, Jena

18. Pfingsten M, Arnold B, Böger A et al (2019) Sektorenübergreifende interdisziplinäre multimodale Schmerztherapie. Schmerz 33:191-203

19. Sachse J, Harke G, Linz W (2012) Extremitätengelenke. Elsevier, München

20. Simmel S, Müller W-D, Bork $H$ et al (2018) Anforderungen an Einrichtungen der Phase C der Traumarehabilitation - Überregionale Traumarehabilitationszentren in der postakuten Rehabilitation. Phys Med Rehab Kuror 28:282-286

21. Stibenz C (2004) Klinische Assessments craniomandibulärer Dysfunktionen (CMD). https://www. $\mathrm{db}$-thueringen.de/servlets/MCRFileNodeServlet/ dbt_derivate_00003866/Diss.txt. Zugegriffen: 09.09.2019

22. Wikipedia (2019) Blockierung (Manuelle Medizin). https://de.wikipedia.org/wiki/Blockierung_ (Manuelle_Medizin).Zugegriffen:09.09.2019

23. Wirtz P, Baumann FT (2018) Bewegungstherapeutische Interventionen in der Onkologie - eine Struktur-und Angebotsanalyse der onkologischen Rehabilitationskliniken in Deutschland. Phys Med Rehab Kuror 28:347-357

24. Zech A, Hübscher M (2012) Sensomotorisches Training zur Prävention von Sprunggelenksverletzungen. Dtsch ZSportmed 63:5-8

25. Zech A, Hubscher $M$, Vogt $L$ et al (2010) Balance training for neuromuscular control and performance enhancement: a systematic review. JAthl Train 45:392-403

In eigener Sache

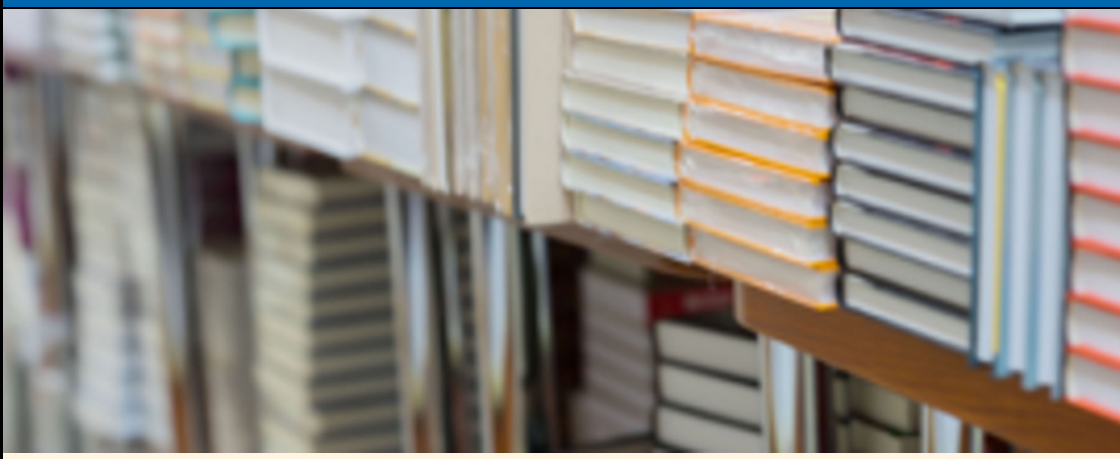

Springer Nature stellt wichtige Lehrbücher während der CoronaKrise kostenfrei zur Verfügung

\section{Lehrbuchpaket zur Intensivmedizin und Pneumologie für pflegerisches und medizinisches Fachpersonal}

Der Ausbruch des Coronavirus bedeutet nachhaltige Einschränkungen für den wissenschaftlichen Lehrbetrieb. Um die nachteiligen Auswirkungen zu lindern, startet Springer Nature ein globales Programm zur Unterstützung des Lernens und Lehrens in Hochschuleinrichtungen weltweit. Lehrende und Studierende aus akademischen Einrichtungen können kostenlos auf mehr als 500 wichtige englisch- und deutschsprachige Lehrbücher von Springer Nature zugreifen. Diese Bücher sind voraussichtlich bis mindestens Ende Juli über die Plattform SpringerLink zugänglich (URL: siehe „Weitere Informationen" am Ende dieser Mitteilung).

Springer Nature hat bereits Tausende von Forschungsartikeln, Büchern und Buchkapiteln zum Thema Coronavirus frei verfügbar gemacht, um den Zugang zu wichtigen Forschungsergebnissen zu erleichtern. Bibliothekare werden unterstützt, wenn sie Mitarbeitern und Studenten einen OnlineZugriff von zuhause ermöglichen möchten. Diese neue Lehrbuch-Initiative zielt darauf ab, das Lernen und Lehren effizient zu unterstützen. Studenten, Akademiker und Universitätsmitarbeiter haben nun freien Zugang zu wichtigen Springer Nature-Lehrbüchern.

Niels Peter Thomas, Managing Director Springer Nature Books: „Angesichts der zunehmenden globalen Auswirkungen der CoronaKrise ist der schnelle Fernzugriff auf Bildungsressourcen unerlässlich geworden. Wir möchten Dozenten, Lehrer und Studenten in dieser herausfordernden Zeit unterstützen und hoffen, dass wir mit der kostenlosen
Freischaltung von über 500 wichtigen Lehrbüchern dazu einen wichtigen Beitrag liefern. An dieser Stelle möchte ich mich für die großartige Unterstützung durch unsere Autoren und Autorinnen bedanken, ohne deren $\mathrm{Zu}$ stimmung wir diese Bücher nicht so ohne weiteres hätten freischalten können."

Ein Buchpaket mit deutschsprachigen Lehrund Fachbüchern für pflegerisches und medizinisches Fachpersonal aus den Gebieten Intensivmedizin und Pneumologie wird ebenfalls auf der Plattform SpringerLink freigeschaltet. Dies ist ein kostenloses Angebot für klinische Einrichtungen in der aktuellen Ausnahmesituation: Der Wissenschaftsverlag möchte Krankenhäusern dabei helfen, sein Personal so fortzubilden, dass es ausreichend qualifiziert ist, um die vermehrt erwarteten Corona-Patienten mit schwerem Verlauf kompetent zu versorgen.

\section{Weitere Informationen:}

Zugang zu den freigeschalteten Lehrbüchern (alle Disziplinen):

www.springernature.com/gp/librarians/newsevents/all-news-articles/industry-newsinitiatives/free-access-to-textbooks-for-institutions-affected-by-coronaviru/17855960

Übersicht aller freigeschalteten Zeitschriftenartikel, Kapitel und Bücher zur CoronavirusForschung: www.springernature.com/de/researchers/ campaigns/coronavirus

\section{Zur Plattform SpringerLink: link.springer.com}

